\title{
Influencing the gradient of material properties by gradation extrusion
}

\author{
Markus Bergmann ${ }^{1, a}$, Andreas Sterzing ${ }^{1, b}$ and Dirk Landgrebe ${ }^{1,2, c}$ \\ ${ }^{1}$ Fraunhofer Institute for Machine Tools and Forming Technology IWU, Reichenhainer Str. 88, \\ 09126 Chemnitz, Germany \\ ${ }^{2}$ Institute for Machine Tools and Production Processes IWP, Technische Universität Chemnitz, \\ Reichenhainer Str. 70, 09126 Chemnitz, Germany ${ }^{3}$ \\ amarkus.bergmann@iwu.fraunhofer.de, bandreas.sterzing@iwu.fraunhofer.de, \\ cdirk.landgrebe@iwu.fraunhofer.de
}

\section{Keywords: Forming, Metal, Severe Plastic Deformation}

\begin{abstract}
Designing material characteristics by grain refinement using Severe Plastic Deformation (SPD) is an attractive way to create outstanding material properties. This paper presents a unique method which combines SPD and impact extrusion. The extrusion die is designed to create additional material deformation to a defined depth, resulting in a gradient from ultra-fine grained to coarse grained microstructure. Due to the large gradient the method is called gradation extrusion. The paper presents a new analytical calculation method and a numerical evaluation of the strain, showing the relationship between tool design and achievable effects and provides initial experimental results.
\end{abstract}

\section{Introduction}

Severely deformed materials can exhibit excellent properties based on the microstructure with grain sizes below one micrometer. A range of forming processes has been developed which allows severe deformation of materials. Some of these Severe Plastic Deformation (SPD) processes subject the whole component volume to severe plastic deformation, for instance Equal Channel Angular Pressing (ECAP) or High Pressure Torsion (HPT), which are reviewed in [1]. Another group of processes called Surface Mechanical Attrition treatment generates a severe deformation in only a thin surface layer up to $50 \mu \mathrm{m}$ [2].

Most SPD methods are limited to laboratory investigations due to technical and economic factors. Currently, there are few low volume niche market products, e.g. sputtering targets [1]. The integration of severe plastic deformation into conventional manufacturing chains is impeded by low productivity, high technology demands, low material utilization or unknown processing characteristics. For example, the ECAP process is widely considered as the SPD method with the highest application potential [3], nevertheless no significant industrial exploitation exists.

The approach presented in this paper provides a contribution to cope with the limiting factors. The unique SPD method of gradation extrusion is based on an impact extrusion process. Extrusion processes are widely applied and they exhibit appropriate characteristics with respect to the requirements of a SPD process. The process principle can be implemented in a large variety of applications regarding machines and equipment. Due to the large gradient of the deformation the process is called gradation extrusion. A major feature of the gradation extrusion principle is the generation of a graded material structure, combining different microstructures within one component. This paper describes the fundamentals of the process, presents calculation of the indicative effective plastic strain, and the verification of the material properties.

The presented investigation compares different gradation extrusion geometries and examines their influence on the effective strain and hardness. The microstructure evaluation by EBSD analysis is presented. 


\section{Process principle and experimental setup}

Gradation extrusion, Fig. 1 (a), is a bulk metal forming method which combines a conventional impact extrusion process and severe plastic deformation of the surface peripheral layer.

The method has initially been designed to process rod-shaped billets. Within the billet a large gradient of severe plastic deformation is created, generating a large gradient of the grain size [4]. In the peripheral layer near the surface grain sizes below one micrometer can be achieved, Fig. 1 (b).

a)

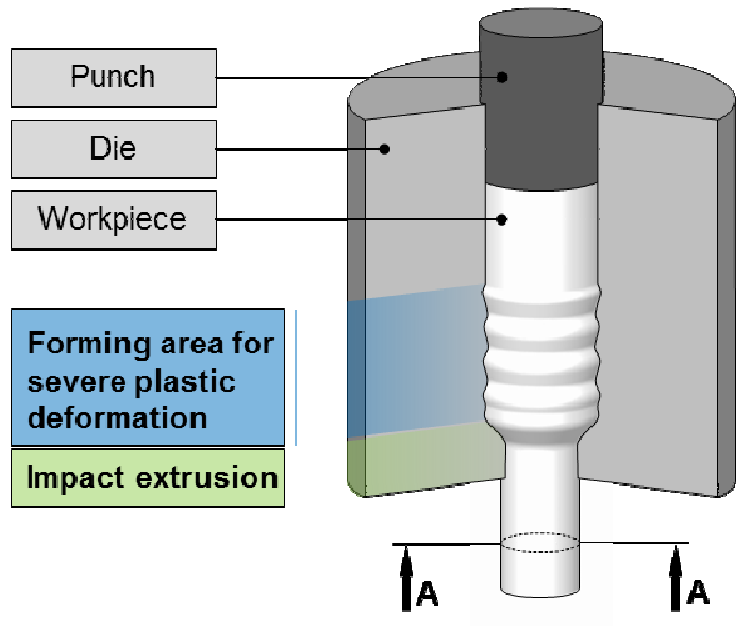

b)

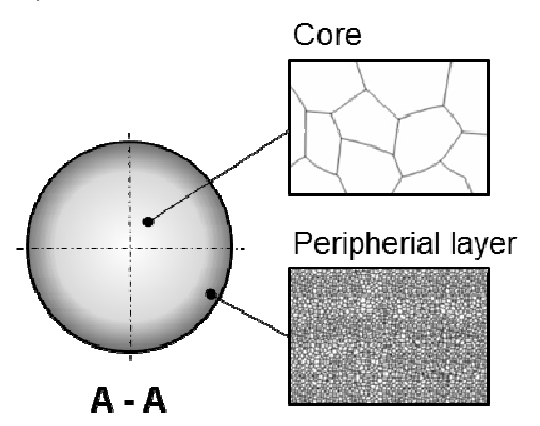

Figure 1: Gradation extrusion principle, adapted from [5]

To induce severe plastic deformation a repeated deformation of the material has to be realized with changing forming direction. This is realized by using a linear moving punch for pressing the workpiece through a special die. The design of the die includes different sections providing the specific forming conditions.

The die sections shown in Fig. 1 (a) create the alternating deformation by reducing and expanding the diameter of the workpiece. The geometry of this severe plastic deformation area consists of SPD forming elements. Typically, several of these elements are combined or stacked. The forming elements are comparatively small in relation to the overall tool size, which limits the severe deformation to a volume of material in proximity to the surface. Among other processing parameters, especially the size, shape and number of the forming elements in relation to the maximum diameter influence and determine deformation characteristics such as the direction of material flow, the intensity and gradation of the severe deformation and the volume portion or layer thickness of the severely deformed material. Generally, the SPD forming elements can be designed relatively free. To create a smooth material flow a geometry with a triangle as basic shape has been chosen for the initial investigation as shown in Fig. 2 (a).

a)

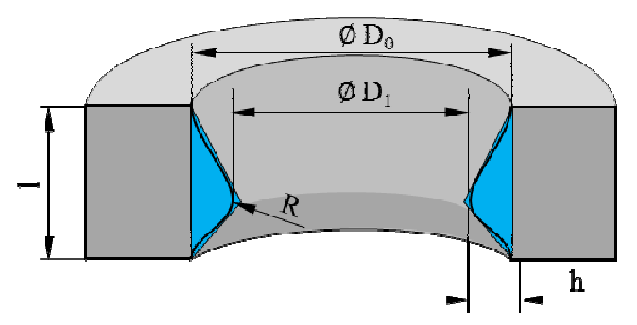

b)

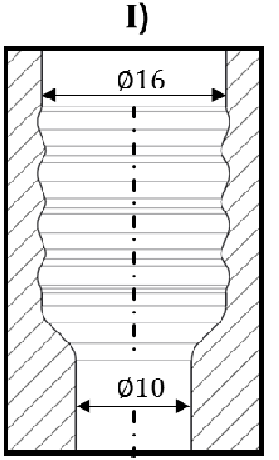

II)

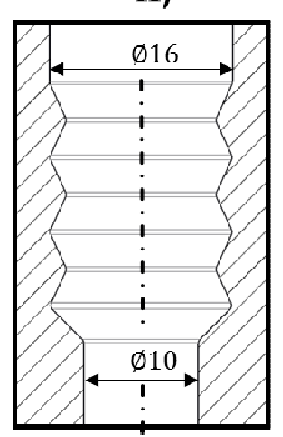

Figure 2: Single SPD forming element (a), investigated gradation extrusion die geometries (b) 
Characteristic geometrical features of a single SPD forming element are the maximum diameter $D_{0}$, the minimum diameter $D_{l}$, the length of the forming element $l$ and the radius $R$. The height of the forming element $h$ is defined by $D_{0}$ and $D_{l}$.

The design of each SPD forming element is combined with the overall function of the die. The final section of the die, i.e. the impact extrusion section, is essential to produce a material flow restriction creating a compressive stress state in the material, which is necessary for complete filling of the cavities between the SPD forming elements. Based on the theoretical considerations of gradation extrusion an experimental set-up was developed [6]. Two different gradation extrusion tool geometries (I, II) have been designed, Fig. 2 (b). Both reduce a bar of $16 \mathrm{~mm}$ to a diameter of $10 \mathrm{~mm}$. The angular geometry (II) is designed and investigated in comparison to the smoothed geometry (I). The new angular geometry (II) with small radii of $0.5 \mathrm{~mm}$ is designed to create ECAP-like deformation.

The gradation extrusion tool is a divided die with two halves in order to allow an opening. The possibility to open the die is needed to remove specimen and provides additional options to study the process and material properties. The experimental tests have been executed on a hydraulic press. The press force is used to keep the die halves closed during the process. A separate punch which is connected to a hydraulic cylinder is used for forming the material.

In the test series the Aluminum alloy EN AW-6082 (AlMgSi1) was used. In the original state the material is in artificially aged condition. The material has been solution heat-treated at $530{ }^{\circ} \mathrm{C}$ and quenched before processing. The material is processed immediately after cooling at room temperature of $20^{\circ} \mathrm{C}$. A MoS -based dry lubricant is applied in order to reduce friction.

The results of the first experiments confirm the basic feasibility of the process for the smoothed (I) and the angular (II) die geometries. The process was studied by observation of intermediate states of the forming operation. The material flow during the process corresponds to the simulated process. Complete filling of the cavities between the SPD forming elements could be observed.

The following section presents results of process simulations using the gradation extrusion geometries in comparison to the basic process principle of impact extrusion.

\section{Numerical simulation of the process}

Fig. 3 shows the numerical simulation of a conventional impact extrusion (III) and two gradation extrusion processes (I), (II). The arrow indicates the extrusion direction. The figures show the quasi-static process state. The effective strain values are plotted. The numerical simulations have been carried out using the software Simufact Forming.

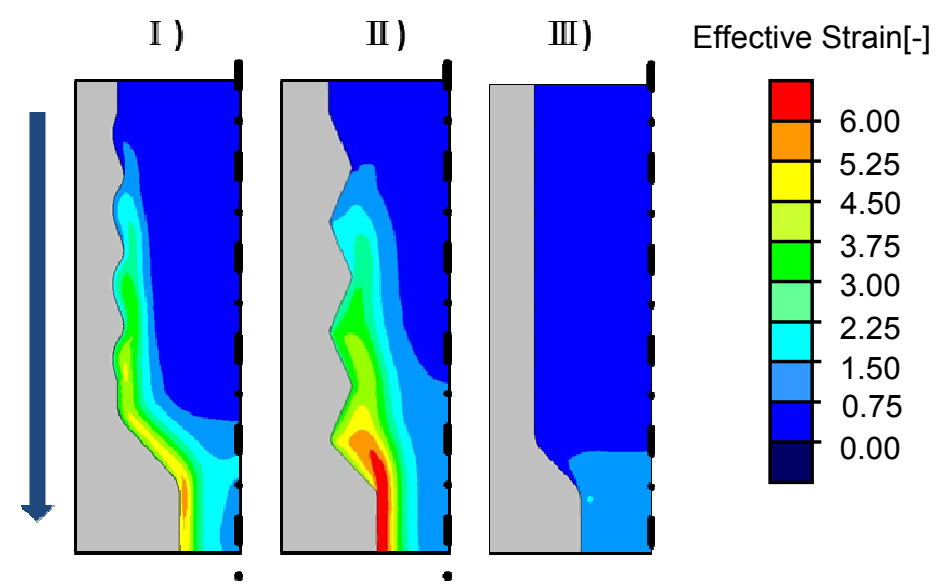

Figure 3: Simulation of the effective plastic strain in the quasi-static state of gradation extrusion (I and II) compared to impact extrusion (III)

The conventional impact extrusion process (III) shows a relatively homogeneous strain distribution of the completely processed material. A determination of the effective strain values 
displays a slight gradient of values from 1 in the core to 1.4 in the near surface area. The gradation extrusion smooth geometry and the angular geometry show an equivalent strain value of approx. 1 on the processed core material. At a distance of approx. $1.5 \mathrm{~mm}$ from the surface a strong increase of the strain occurs in (I) and (II). Maximum levels of 5.3 and 6.9 were calculated with the smooth design (I) and with the angular design (B), respectively, in the surface area.

Furthermore the process simulations of the two gradation extrusion versions show that every single forming element leads to additional deformation, increasing the effective strain.

\section{Analytical calculation of strain}

For the analytical calculation of the strain values applied to the peripheral layer the forming process can be divided into two main mechanisms. During the forming process the billet diameter is changed several times. An alternating reduction and expansion occurs.

The first mechanism consists of extrusion steps. The common effective strain calculation of one extrusion step is

$$
\varphi_{v I E}=\ln \left(1+\varepsilon_{A}\right)
$$

with a reduction of cross-section

$$
\varepsilon_{A}=\frac{D_{1}^{2}-D_{0}^{2}}{D_{0}^{2}} \text {. }
$$

The second mechanism generates a deformation in the peripheral near-surface area which is similar to the ECAP process. In Fig. 4 the principle is depicted based on the angular geometry (II). In the near-surface area an ECAP channel is assumed with several consecutive ECAP-steps which are numbered 1 to 8 .

In contrast to actual ECAP processes, the gradation extrusion process creates the shearing in a channel with a certain width. Only one side of the channel is a solid die. For this reason a symmetrical channel is assumed with width $b$, angle $\phi$ and radii $R$.

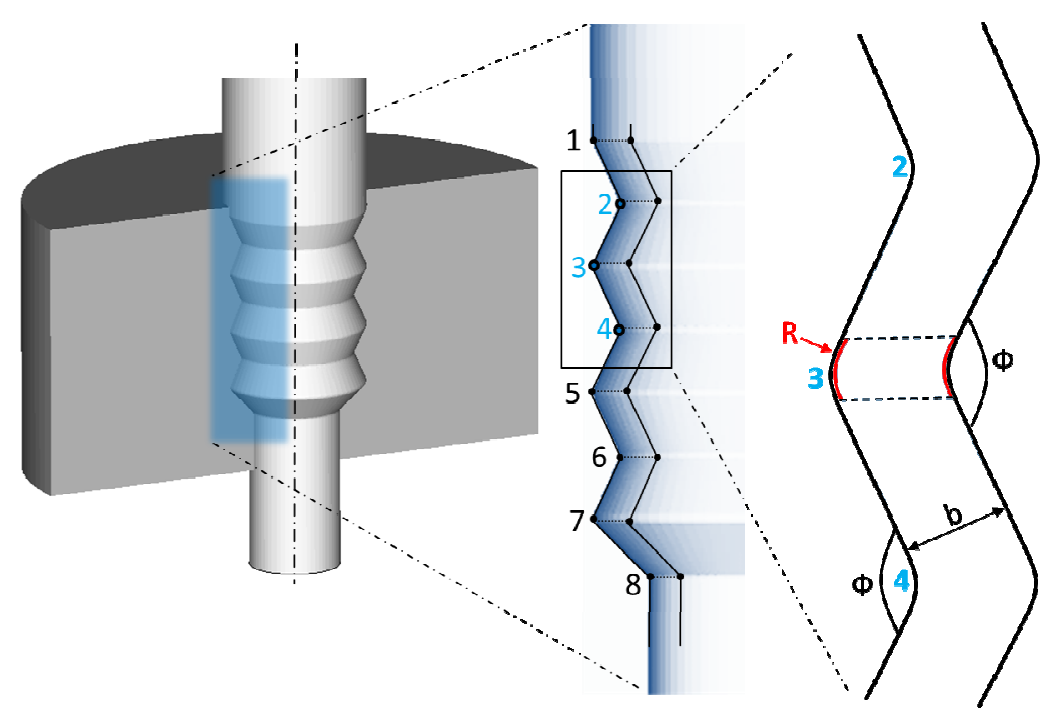

Figure 4: ECAP-like deformation in peripheral area within the gradation extrusion die

Different approaches exist for calculating the strain of ECAP processes, such as shown by Segal [7], Iwahashi [8] or Pérez [9]. The results of Pérez [9] with a shearing angle of $0^{\circ}$ can be 
combined with the strain calculation presented by Segal [7] to calculate the effective strain for each step with the following formula:

$$
\varphi_{v E C A P}=\frac{2}{\sqrt{3}} \cdot \cot \left(\frac{\phi}{2}\right)
$$

The resulting effective strain is calculated by summing up the values of every single ECAP-like forming step and every single impact extrusion step

$$
\varphi_{v}=\sum\left|\varphi_{i_{-} v I E}\right|+\sum\left|\varphi_{i_{-} v E C A P}\right|
$$

The calculated value represents the die with a channel near the forming elements or the severely deformed area with a specified width.

In the investigated angular die geometry (II) the effective strain value is calculated by summing up the reduction, upsetting and shearing steps. Table 1 shows the calculated values of the extrusion and the ECAP-like deformation for the angular die (II) shown in Fig. 4.

\begin{tabular}{|c|c|c|c|c|}
\hline $\begin{array}{c}\text { Element } \\
i\end{array}$ & $\begin{array}{c}\text { Diameter Change } \\
D_{1}-D_{0}\end{array}$ & $\begin{array}{l}\text { Eff. Strain Extrusion } \\
\qquad \varphi_{i_{-}} v I E\end{array}$ & $\begin{array}{c}\text { Channel Angle } \\
\phi\end{array}$ & $\begin{array}{l}\text { Effective Strain ECAP } \\
\qquad \varphi_{i}{ } v E C A P\end{array}$ \\
\hline 1 & - & & $155.2^{\circ}$ & 0.25 \\
\hline 2 & $\emptyset 16 \rightarrow \emptyset 13$ & -0.415 & $130.5^{\circ}$ & 0.53 \\
\hline 3 & $\emptyset 13 \rightarrow \emptyset 16$ & 0.415 & $130.5^{\circ}$ & 0.53 \\
\hline 4 & $\emptyset 16 \rightarrow \emptyset 13$ & -0.415 & $130.5^{\circ}$ & 0.53 \\
\hline 5 & $\emptyset 13 \rightarrow \emptyset 16$ & 0.415 & $130.5^{\circ}$ & 0.53 \\
\hline 6 & $\emptyset 16 \rightarrow \emptyset 13$ & -0.415 & $130.5^{\circ}$ & 0.53 \\
\hline 7 & $\emptyset 13 \rightarrow \emptyset 16$ & 0.415 & $110.2^{\circ}$ & 0.80 \\
\hline 8 & $\emptyset 16 \rightarrow \emptyset 10$ & -0.94 & $135.0^{\circ}$ & 0.47 \\
\hline & $\sum \mid \varphi$ & $=3.4$ & & $C A P \mid=4.2$ \\
\hline Total & \multicolumn{4}{|c|}{$\varphi_{v}=7.6$} \\
\hline
\end{tabular}

Table 1: Calculated strain values for the angular gradation die (II)

The calculated equivalent strain value within the severely deformed near-surface material volume is 7.6. This value generally corresponds to the maximum value of 6.9 obtained by numerical simulation. The lower value for the numerical simulation can be explained by the fact that no hardening effect is taken into account in the analytical calculation.

Using the introduced strain calculation, the proportion of ECAP-like deformation could be estimated. In the case of the angular die geometry (II) the ECAP-like deformation dominates with a value of 4.2 .

\section{Evaluation of graded material properties}

The effect of gradation extrusion on material properties has been analyzed by hardness measurements and a microstructure evaluation using EBSD analysis. Increased hardness values are an indicator for the intensity of the severe plastic deformation. Two approaches were performed to measure hardness distribution.

The hardness distribution map, Fig. 5 (a), presents the measured values using a specimen formed with the smoothed die (I), in order to obtain information on the local distribution of hardness 
between the unformed and completely formed states of the material. The sample is a longitudinal cross-section made of a specimen retrieved at an intermediate stage during forming. The crosssection contains an area of material before forming, a material section in the forming area including the SPD forming elements, and a section of material after forming. The hardness value map is superimposed on the specimen cross-section, Fig. 5 (a).

a)

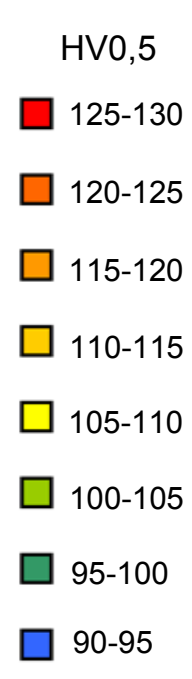

b)

\section{b1)}

First SPD forming element

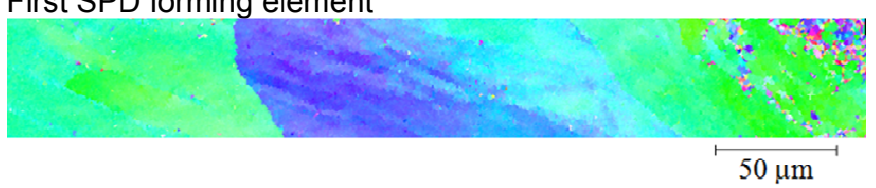

b2)

Third SPD forming element

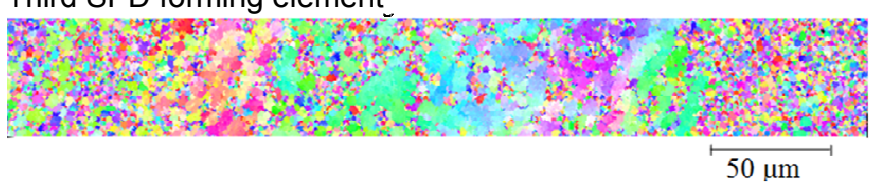

Figure 5: Hardness distribution (a) and the microstructure near the surface in sample processed by gradation extrusion (b) [4]

In the hardness distribution map increasing values can be observed near the contact with the SPD forming element. Consecutively, with additional forming elements, the hardness continues to increase and reaches its maximum after the final extrusion step. In the SPD forming element section the hardness increases significantly only in the peripheral layer and reaches a depth up to approx. $1.5 \mathrm{~mm}$ from the surface.

Overall, two gradients can be detected: (1) increasing hardness from the initially unformed material towards the completely formed final material state, and (2) increasing hardness from the core of the specimen (middle axis) towards the outer surface.

The hardness in the severely deformed near-surface area is increased from approx. 95 HV0.5 in the core to $125 \mathrm{HV} 0.5$. The gradient after final processing ranges from $105 \mathrm{HV} 0.5$ in the core to $125 \mathrm{HV} 0.5$ at a distance of $0.2 \mathrm{~mm}$ from the surface.

To characterize the microstructure an EBSD analysis was carried out. Two positions indicated in Fig. 5 (a) are presented in Fig. 5 (b). The detected Aluminum structure at the first forming element, Fig. 5 (b1), showed a normal, coarse-grained microstructure. A grain size larger than $50 \mu \mathrm{m}$ can be observed. The material structure at position (b2) has been fundamentally changed in comparison to (b1). Sub-structuring and reorientation of the grain can be detected. Within the characterized area with a width of $500 \mu \mathrm{m}$ the grain size distribution is inhomogeneous. A large ratio of small grains in a range of 1 to $2 \mu \mathrm{m}$ is created with high angle boundaries. Furthermore fragments with grain sizes larger than $10 \mu \mathrm{m}$ can be observed. Even though the grain shape and size is not homogeneous, the typical effect of severe plastic deformation of grain refinement with numerous high angle boundaries can be detected clearly.

The presented EBSD analysis illustrates the effect of gradation extrusion on the material microstructure. The elaborate evaluation of gradation extrusion requires further microstructure analysis.

In a second hardness evaluation approach a comparison has been made on the effect of the two different die geometries (I) and (II) on hardness. For this purpose the hardness distribution was 
measured along the cross-sections of the formed specimens. The two specimens formed with the two dies are shown in Fig. 6. The material of these samples shows no visible damage.

The diagram in Fig. 6 presents the hardness measurement values for the periphery $(0=$ surface $)$ to the core ( $\mathrm{R}=8 \mathrm{~mm}$, at center). The first measuring point is at a distance of $0.3 \mathrm{~mm}$ to the surface. The lower data series are measured on the samples before the material is significantly deformed. The two higher data series show the hardness measurement of the completely deformed material.

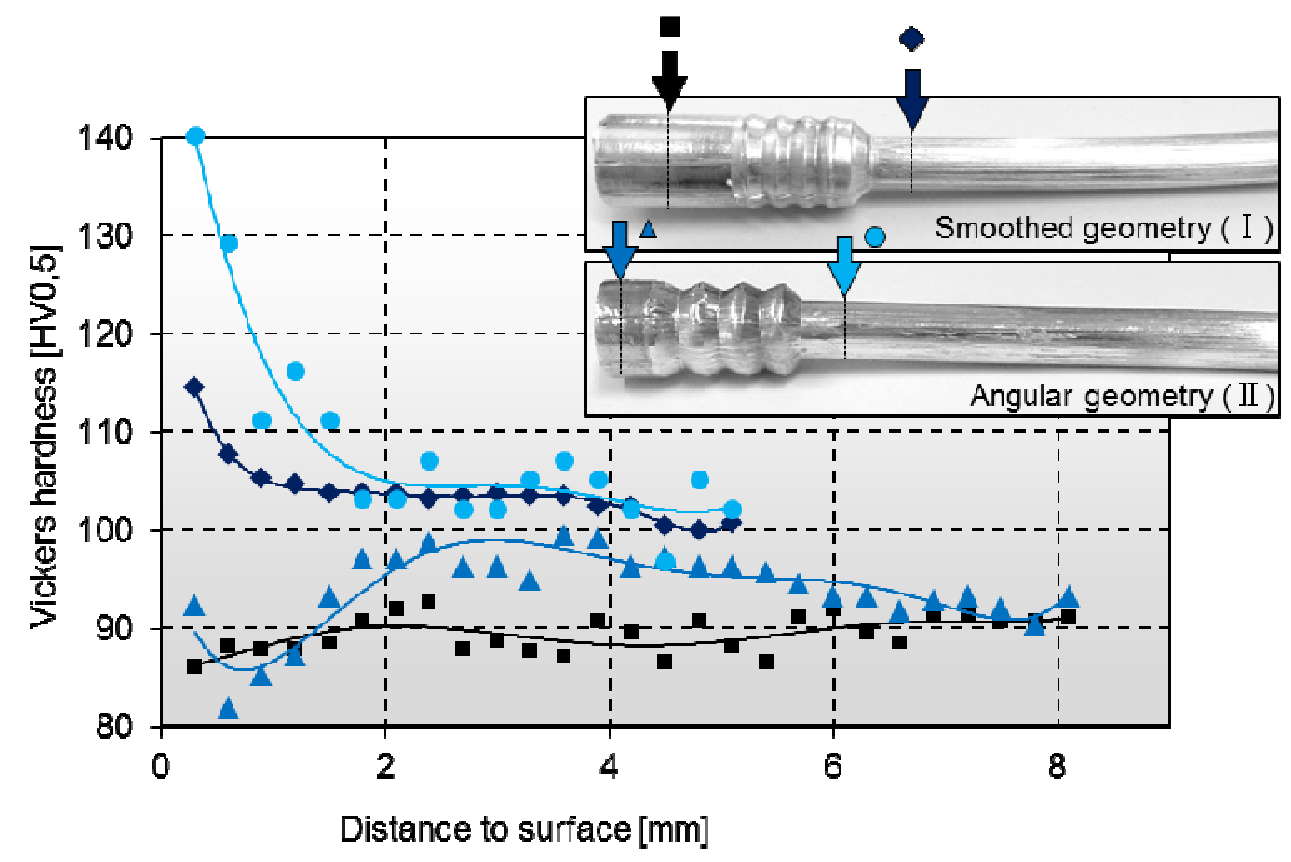

Figure 6: Measurement of hardness distribution with the two specimens after gradation extrusion

The measured distributions demonstrate that both samples show hardening effects on the complete cross-section. Significant hardening can be observed in the near-surface area. The intensive plastic deformation caused by the forming elements leads to hardening which is created with both die geometries. Maximum hardness values reached are 115 HV0.5 using the smooth geometry and 140 HV0.5 on the sample formed with the angular die. Compared to the average hardness of the initial condition of $90 \mathrm{HV} 0.5$, a relatively strong hardening effect can be generated in the near-surface or peripheral area.

The comparison of the hardness difference between the samples made with the different die geometries corresponds to the results of the numerical simulation of effective plastic strain presented in section 3.

The hardening behavior shows that the developed geometry of the SPD forming elements is appropriate to control the severe material deformation.

The presented data displays initial analytical and experimental results. Further EBSD analysis is required as well other approaches to determine material characteristics and mechanical properties.

\section{Summary and conclusion}

The unique SPD method of gradation extrusion is presented and investigated. Two different gradation die geometries were examined. The process simulation reveals the generation of a much higher strain in local volumes compared to impact extrusion, and the geometry of the SPD forming elements considerably affects the strain.

An analytical calculation approach is presented, taking into account a combination of extrusion and shearing in the near-surface area. As an example a calculation for the specified angular die geometry is carried out. The calculation can be used, e.g. to analyze the effects of die geometry on effective plastic strain or for comparison with results of the numerical simulation. 
The experimental investigations of the process were carried out using two different die geometries. Using SPD forming elements in the tools, a grain refinement can be achieved with a range of 1 to $2 \mu \mathrm{m}$. Mechanical properties were analyzed using hardness measurement. The resulting increase in hardness and the gradient of hardness correspond to the results of the FE simulation. An EBSD analysis showed the effect of gradation extrusion on the microstructure in the near-surface area of the formed material.

Using this process principle, the opportunity is provided to realize the integration of a SPD operation into forming process chains in a relatively effortless manner. Possible applications of the gradation extrusion principle are Aluminum bolts. The application could be furthermore expanded to the manufacturing of Titanium implants where work hardening can be used to achieve significant increase in strength of pure materials.

Further developments will focus on the understanding of the interaction between the geometry of SPD forming elements and the resulting microstructure and mechanical properties.

\section{Acknowledgements}

The authors acknowledge the Deutsche Forschungsgemeinschaft (DFG) for supporting this work carried out within the framework of Collaborative Research Centre 692. Furthermore the authors thank Dagmar Dietrich of the Institute of Material Science and Engineering at Technische Universität Chemnitz for supporting the EBSD analysis.

\section{References}

[1] A. Azushima, R. Kopp , A. Korhonen, D. Yang, F. Micari, G. Lahoti, P. Groche, J. Yanagimoto, N. Tsuji, A. Rosochowski, A. Yanagida, Severe plastic deformation (SPD) processes for metals CIRP Annals - Manufacturing Technology, 57 (2008) 716 - 735.

[2] K. Lu, L. Shaw, Bulk Materials with a Nanostructured Surface and Coarse-grained Interior, Zehetbauer, M. J. Zhu, Y. T. (ed.) Bulk Nanostructured Materials, Weinheim Wiley-VCH (2009) $649-671$.

[3] Y. T. Zhu, T. G. Langdon, The fundamentals of nanostructured materials processed by severe plastic deformation, JOM Journal of the Minerals, Metals and Materials Society 56/10 (2004) $58-63$.

[4] M. Bergmann, Verfahren zur Herstellung gradiert hochgradig plastisch umgeformter Werkstoffe, Auerbach, Verl. Wiss. Scripten (2013)

[5] R. Neugebauer, M. Bergmann, Local Severe Plastic Deformation by Modified Impact Extrusion Process, Steel Research International - Special Edition: 14th International Conference on Metal Forming 2012 - in Krakau (2012) 471 - 474.

[6] R. Neugebauer, A. Sterzing, R. Selbmann, R. Zachäus, M. Bergmann, Gradation extrusion Severe plastic deformation with defined gradient, Materialwissenschaft und Werkstofftechnik 43/7 (2012) $582-588$.

[7] V. Segal, Materials processing by simple shear Materials Science and Engineering 197 (1995) $157-164$.

[8] Y. Iwahashi, J. Wang, Z. Horita, M. Nemoto, Langdon TG, Principle of Equal-channel Angular Pressing for the Processing of Ultra-fine Grained Materials. Scripta Materialia 35/2 (1996) 143146.

[9] C. J. L. Pérez, Upper bound analysis and FEM simulation of equal fillet radii angular pressing Modelling and Simulation, Materials Science and Engineering 12 (2004) 205. 